Original

\title{
Valoración de hábitos de vida saludables de los trabajadores expuestos cancerígenos laborales de la provincia de Salamanca
}

\author{
Analysis of the healthy lifestyles of workers exposed to occupational \\ carcinogens in the province of Salamanca
}

\section{Jesús González Sánchez}

DUE. Especialista en Enfermería del Trabajo

Servicio de Prevención de Riesgos Laborales

MC -Prevención Salamanca. España

Recibido: 30-01-14

Aceptado: 05-06-14

\section{Correspondencia}

Jesús González Sánchez

MC - Prevención Salamanca

Plaza Mariseca, s/n. esquina Calle del Hornazo

37003 - Salamanca. España

Teléfono móvil: 630789181

E-mail: igonzalezsa@mc-prevencion.com

Resumen

Objetivos: Conocer los hábitos de vida de los trabajadores cubiertos por los servicios de prevención ajenos de la provincia de Salamanca. Comparar grupos de trabajadores expuestos y no expuestos a cancerígenos laborales.

Método: Se ha realizado un estudio epidemiológico observacional, de carácter descriptivo transversal. La selección de la muestra fue mediante muestreo aleatorio estratificado en los trabajadores pertenecientes a los servicios de prevención ajenos de la provincia de Salamanca. Las variables recogidas mediante cuestionario: características sociodemográficas, de exposición a cancerígenos laborales y hábitos de vida, utilizando como fuente de información las historias clínico-laborales.

Resultados: Un 32\% de los 835 trabajadores estudiados se encontraba expuesto a algún cancerígeno laboral. Los trabajadores expuestos a cancerígenos y que pertenecen al sector servicios refieren un mayor hábito tabáquico $(44,7 \%)$, sin embargo, los trabajadores no expuestos y que pertenecen al sector agricultura y ganadería refieren un mayor consumo de alcohol (58,8\%). En global, los trabajadores no expuestos a cancerígenos realizan de forma habitual una dieta equilibrada $(74,6 \%)$ en comparación con los trabajadores expuestos (61,8\%). La realización de actividad física es similar en ambos grupos de trabajadores.

Conclusiones: La exposición a cancerígenos laborales es un factor que puede influir en ciertos hábitos de vida en los trabajadores, como en el hábito tabáquico, el consumo de alcohol y la realización de dieta equilibrada de forma habitual.

Med Segur Trab (Internet) 2014; 60 (235) 344-357

Palabras claves: Salud laboral, Enfermedades profesionales, Cáncer laboral, Factores de riesgo, Servicios de prevención. 


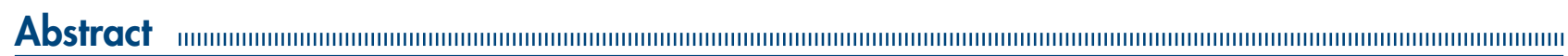

Objectives: Knowing the lifestyles of those protected by external prevention services in the province of Salamanca workers. Compare groups of workers exposed and not exposed to occupational carcinogens.

Methods: Observational epidemiological survey was conducted descriptive cross. The selection of the sample was stratified random sampling by workers belonging to prevention services outside of the province of Salamanca. Variables collected by questionnaire: sociodemographic characteristics, exposure to occupational carcinogens and lifestyle, using as a source of clinical information labor stories.

Results: $32 \%$ of the 835 workers studied was exposed to some occupational carcinogen. Workers exposed to carcinogens and that belong to the service sector reported a higher smoking (44.7\%), however, the non-exposed workers who belong to the agriculture and livestock sector, reported a higher consumption of alcohol (58.8\%). In total, workers not exposed to carcinogens usually perform a balanced (74.6\%) diet compared to exposed workers (61.8\%). Performing physical activity is similar in both groups of workers.

Conclusions: Exposure to occupational carcinogens is a factor that can influence certain lifestyle habits among workers, as in smoking, drinking and making balanced diet regularly.

Med Segur Trab (Internet) 2014; 60 (235) 344-357

Keywords: Occupational bealth, occupational diseases, labor cancer, risk factors, prevention services. 


\section{INTRODUCCIÓN}

Los procesos cancerosos son el resultado de la interacción del individuo y su entorno, o lo que es lo mismo, la interacción entre factores genéticos y factores externos al individuo, pero estrechamente relacionados con él como son: la dieta, hábitos tóxicos (tabaco y alcohol), exposiciones laborales y/o ambientales ${ }^{1-4}$.

Existen trabajos en los que se analizan hábitos de vida de los trabajadores y como influyen ciertas características relacionadas con su trabajo, como la realización de trabajos a turnos ${ }^{5,6}$, el estrés ${ }^{7}$, o trabajos relacionados con producción de comidas ${ }^{8}$. Otros estudios que tratan sobre el cáncer laboral, se centran en cambio en la exposición a sustancias nocivas ${ }^{9}$, pero apenas existen estudios que analicen los hábitos de vida de los trabajadores expuestos y su posible relación con dicha exposición.

Además debemos tener en cuenta, que hábitos de vida no saludables como el hábito de fumar, pueden aumentar el efecto nocivo de ciertos cancerígenos laborales, como la exposición al amianto, sílice, ciertos plaguicidas y disolventes.

Por todo lo anterior, el objetivo de este trabajo es conocer las diferencias que hay en los hábitos de vida saludables de la población trabajadora de la provincia de Salamanca, protegida por servicios de prevención ajenos, en función de la exposición a algún cancerígeno laboral, para poder implantar las medidas preventivas encaminadas a promocionar conductas más saludables y evitar la aparición de enfermedades importantes.

\section{MATERIAL Y MÉTODO}

\section{Diseño del estudio}

Se realizó un estudio epidemiológico observacional, de carácter descriptivo transversal, en una muestra de 835 trabajadores pertenecientes a alguno de los 11 servicios de prevención ajenos acreditados en la provincia de Salamanca desde el 1 de enero hasta el 31 de diciembre de 2011 y que se habían realizado su correspondiente examen de salud laboral en las correspondientes unidades básicas de salud (UBS) o en las unidades móviles de salud laboral.

Las edades de los trabajadores estaban comprendidas entre los 18 y 65 años, realizaban su trabajo por cuenta ajena o por cuenta propia, pudiendo pertenecer tanto a la administración pública como a la empresa privada.

Se realizó mediante muestreo aleatorio estratificado por cada servicio de prevención ajeno. El número de sujetos seleccionados ha sido proporcional al número total de trabajadores cubiertos por cada entidad.

\section{Población de estudio}

El tamaño de la muestra se ha calculado para la estimación de proporciones, en una población de trabajadores cubierta por los servicios de prevención ajenos de la provincia de Salamanca de 42367, considerando un riesgo alfa de 0,05 y una precisión del 95\%. Al desconocer la prevalencia esperada de factores de riesgo se ha asumido el $50 \%$, al ser la situación más desfavorable, y el efecto del diseño estimado para el muestreo estratificado es de 2 , por lo que se precisan 762 sujetos, que se seleccionaron mediante muestreo aleatorio simple y se distribuyeron proporcionalmente al tamaño de trabajadores de cada entidad. Para compensar las posibles pérdidas, hemos incrementado la muestra en un $10 \%$, siendo por tanto el número de historias revisadas de 835.

Se excluyeron a los trabajadores con un examen de salud incompleto, o sea que no tenían una descripción adecuada de todos los apartados y cuestionarios necesarios, según establecen los distintos protocolos de vigilancia de la salud aplicados, en función de los riesgos laborales a los que están expuestos los trabajadores. 


\section{Fuente de Información}

Se utilizó como fuente de información las historias clínico-laborales informatizadas pertenecientes a los departamentos de Medicina del Trabajo de cada servicio de prevención ajeno, al constituir el documento que recoge toda la información referente a la vigilancia y control de la salud de los trabajadores. Los contenidos que deben de contener las historias clínico-laborales, se establecen en el Real decreto 39/97 de 17 de enero, por el que se aprueba el Reglamento de los Servicios de Prevención ${ }^{10}$.

\section{Variables estudiadas}

Los apartados de anamnesis de las historias clínico-laborales de los servicios de prevención visitados, nos han aportado la información necesaria para la adecuada cumplimentación de los cuestionarios de nuestro estudio. Las variables recogidas fueron:

- Características generales de la población (edad, sexo y sector de actividad económica al que pertenecen).

- Exposición a algún agente cancerígeno laboral.

Hemos considerado exposición de un trabajador a algún agente cancerígeno laboral, la presencia de al menos un agente, que figure en la clasificación de la Agencia Internacional de Investigación sobre el Cáncer (IARC) ${ }^{11}$, en los riesgos de exposición que figuran en su historia clínico-laboral. La exposición de los trabajadores ante alguno de los factores de riesgo cancerígeno, podrá ser actual o bien anterior, pero siempre dentro del periodo de tiempo estudiado, sin ser condición para la clasificación de trabajador expuesto, de ningún periodo de tiempo mínimo de exposición, así como de cualquier grado de utilización de los equipos de protección individual pertinentes.

- Consumo de tabaco: En la valoración del hábito tabáquico de los trabajadores de nuestro estudio, se han tenido en cuenta tres posibles opciones dentro de este apartado:

- Trabajador "no fumador".

- Trabajador "exfumador", registrando el número de años que fumó.

- Trabajador "fumador", registrando el tipo de tabaco que fuma (cigarrillos, puros o pipa), así como el número de unidades por día.

- Consumo de alcohol: Teniendo en cuenta el informe sobre el alcohol, elaborado por la comisión clínica de la Delegación del Gobierno para el plan nacional sobre drogas, en el que se utilizan las Unidades de Bebida Estándar (UBE) para la clasificación del consumo de alcohol, y en el que se detalla que en Europa se tiende a considerar el límite para establecer un consumo de riesgo en 5 UBEs/ día para el hombre y en 3 UBEs/día para la mujer, se han tenido en cuenta cuatro posibles opciones en este apartado:

- No consumidor.

- Consumidor en el pasado, pero no actualmente.

- Consumidor ocasional, cuando el trabajador refiere consumir menos de 5 unidades de alcohol al día en el caso de los varones y menos de 3 en el caso de las mujeres.

- Consumidor habitual, cuando el trabajador refiere consumir 5 o más unidades de alcohol al día en el caso de los varones y 3 o más unidades de alcohol al día, en el caso de las mujeres.

- Alimentación: En la recogida de datos en el cuestionario, a cerca de la alimentación que lleva a cabo el trabajador, hemos tenido en cuenta dos posibles opciones: dieta equilibrada y dieta no equilibrada, entendiendo por dieta equilibrada, aquella dieta variada que realiza de forma frecuente el trabajador, y que contiene todos los alimentos básicos: pan, cereales, arroz y pasta, como principales aportes de hidratos de carbono; frutas, verduras, hortalizas, como fuente principal de 
vitaminas, fibra y minerales; leche y productos lácteos, como aportes claves de calcio y proteínas animales, y carnes, pescados y huevos, como fuente principal de proteínas de origen animal, tal y como se establece en la guía de alimentación saludable, editada por la Sociedad Española de Nutrición Comunitaria (SENC).

- Actividad física: La valoración de la actividad física, se ha realizado teniendo en cuenta los siguientes valores:

- Sedentario, si el trabajador refiere no realizar ningún tipo de actividad física.

- Activo, valorando dos niveles de actividad física según se realicen menos de 2 horas a la semana, o bien un mínimo de dos horas o más.

Como actividad física se ha tenido en cuenta desde la realización de algún tipo de ejercicio o deporte de forma individual o colectiva, así como la actividad de caminar un mínimo de 20-30 minutos en cada sesión.

La valoración de la actividad física en el trabajador, se ha realizado con independencia del nivel de actividad física que se pueda requerir en su puesto de trabajo, durante su jornada laboral.

- Turnicidad y/o nocturnidad: Hemos seleccionado a los trabajadores con turnicidad, cuando la realización de su horario de jornada laboral implica una organización del trabajo en la empresa a través de la rotación de distintos trabajadores, que ocupan de forma sucesiva los mismos puestos de trabajo, según un cierto ritmo, continuo o discontinuo, implicando para el trabajador la necesidad de prestar sus servicios en horas diferentes en un período determinado de días o de semanas; Los trabajadores seleccionados con nocturnidad, son los que realizan sus tareas entre las 10 de la noche y las 6 de la mañana, según se establece en el Real Decreto Legislativo 1/1995, de 24 de marzo, por el que se aprueba el texto refundido de la Ley del Estatuto de los Trabajadores ${ }^{12}$.

\section{Análisis estadístico}

Se realizó un análisis descriptivo para evaluar el comportamiento individual de las variables, con el género y si están los trabajadores expuestos o no a algún factor de riesgo cancerígeno laboral. Las variables continuas se han expresado con la media \pm desviación estándar para variables con distribución normal, y con la mediana para variables que no seguían distribución normal. Las variables cualitativas se han expresado con número y porcentaje. El análisis de la diferencia de medias entre dos categorías de variables cuantitativas se realizó mediante la prueba de la $\mathrm{T}$ de studen o de Mann-Whitney. El análisis de la variables cualitativas fue mediante el test ji cuadrado o test de Fischer.

El procesamiento de los cuestionarios se realizó con el soporte informático necesario, utilizando en primer lugar el sistema teleform para recoger los datos directamente desde el cuestionario. Para realizar el análisis estadístico se ha utilizado el programa SPSS versión 20. Para los contrastes de hipótesis se ha fijado un riesgo $\alpha$ de 0,05 como límite de significación estadística.

\section{RESULTADOS}

El 61\% (513) de la muestra analizada eran trabajadores varones (Figura 1). La edad media en global fue de 42 años, siendo en mujeres 40 y en varones $43(\mathrm{p}<0,001)$. Los

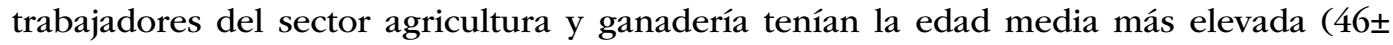
años). Hay un mayor porcentaje de trabajadores varones que realizan turnicidad en su jornada laboral (el $71,2 \%$ frente al 61,5 de las mujeres). Sin embargo, las mujeres trabajadoras refieren realizar mayor nocturnidad. Los sectores de actividad económica con mayor número de trabajadores, corresponden al sector servicios y al sector industria con $366(43,8 \%)$ y $338(40,5 \%)$ trabajadores respectivamente (Tabla I). 
Figura 1. Distribución de los trabajadores por sexos

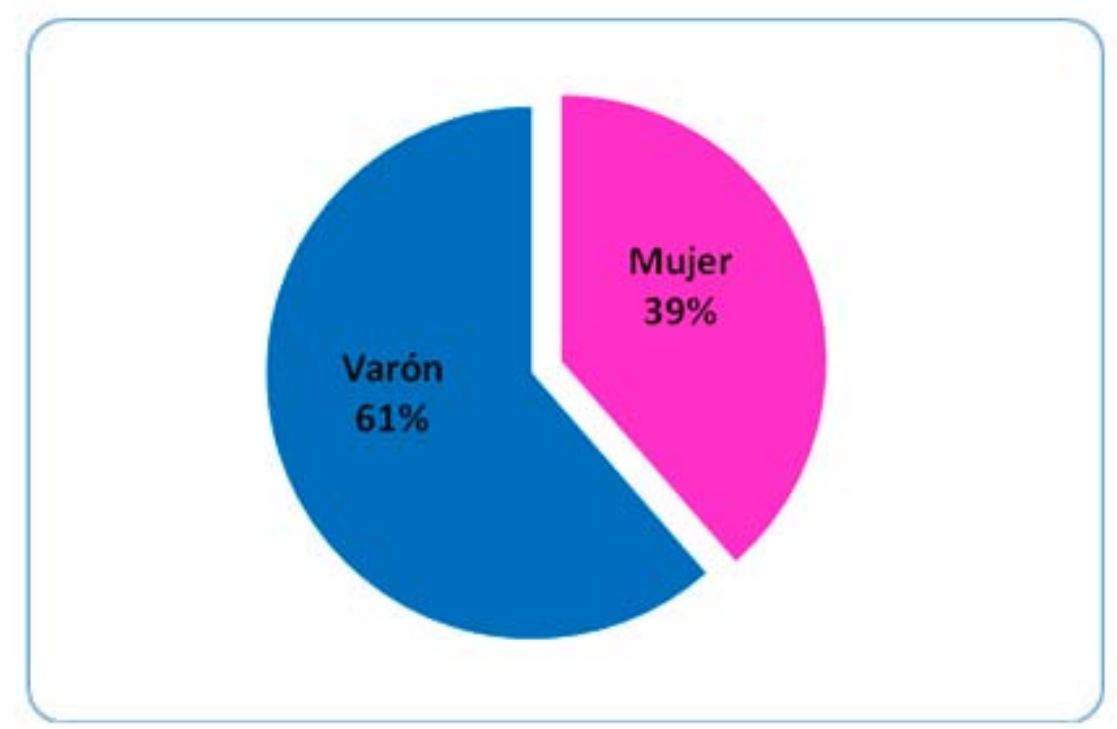

Tabla I. Características generales y por sectores de actividad

\begin{tabular}{|c|c|c|c|c|}
\hline Variable & $\begin{array}{c}\text { Global } \\
\mathrm{n}=\mathbf{8 3 5}\end{array}$ & $\begin{array}{l}\text { Mujeres } \\
\mathrm{n}=322\end{array}$ & $\begin{array}{l}\text { Varones } \\
\mathrm{n}=513\end{array}$ & $\mathbf{P}$ valor \\
\hline \multicolumn{5}{|l|}{ Total } \\
\hline Edad & $42(50-34)$ & $40(48-33)$ & $43(51-35)$ & $<0,001$ \\
\hline Turnicidad, n. ${ }^{\circ}(\%)$ & $563(67,4)$ & $198(61,5)$ & $365(71,2)$ & 0,004 \\
\hline Nocturnidad, n. ${ }^{\circ}(\%)$ & $95(11,3)$ & $41(12,7)$ & $54(10,5)$ & 0,392 \\
\hline Agricultura y Ganadería & $\mathrm{n}=\mathbf{2 5}$ & $\mathbf{n}=\mathbf{1}$ & $\mathbf{n}=\mathbf{2 4}$ & \\
\hline Edad & $46(49-41)$ & $42(42-42)$ & $46(49,5-41)$ & 0,640 \\
\hline Turnicidad, n. $^{\circ}(\%)$ & $1(4,0)$ & $0(0,0)$ & $1(4,2)$ & 0,835 \\
\hline Nocturnidad, n. ${ }^{\circ}(\%)$ & $2(8,0)$ & $1(100)$ & $1(4,2)$ & 0,080 \\
\hline Construcción & $\mathrm{n}=106$ & $\mathbf{n}=\mathbf{2}$ & $n=104$ & \\
\hline Edad & $42(50-32)$ & $34(34-27)$ & $42(50-32)$ & 0,334 \\
\hline Turnicidad, n. ${ }^{\circ}(\%)$ & $2(1,9)$ & $0(0,0)$ & $2(1,9)$ & 0,843 \\
\hline Nocturnidad, n. ${ }^{\circ}(\%)$ & $0(0,0)$ & $0(0,0)$ & $0(0,0)$ & - \\
\hline Industria & $\mathrm{n}=338$ & $\mathbf{n}=\mathbf{8 0}$ & $\mathrm{n}=\mathbf{2 5 8}$ & \\
\hline Edad & $39,5(49-32,75)$ & $35,5(44-30)$ & $40(50-33)$ & 0,003 \\
\hline Turnicidad, n. ${ }^{\circ}(\%)$ & $199(58,9)$ & $41(51,3)$ & $158(61,2)$ & 0,120 \\
\hline Nocturnidad, n. ${ }^{\circ}(\%)$ & $29(8,5)$ & $7(12,5)$ & $22(8,5)$ & 0,536 \\
\hline Servicios & $n=366$ & $\mathrm{n}=239$ & $\mathbf{n}=\mathbf{1 2 7}$ & \\
\hline Edad & $43(50,25-36)$ & $40(49-34)$ & $47(54-38)$ & 0,000 \\
\hline Turnicidad, n. ${ }^{\circ}(\%)$ & $236(64,5)$ & $154(64,4)$ & $82(64,6)$ & 0,998 \\
\hline Nocturnidad, n. ${ }^{\circ}(\%)$ & $64(17,5)$ & $33(13,8)$ & $31(24,4)$ & 0,021 \\
\hline
\end{tabular}

Los valores para datos continuos distribuidos asimétricamente están expresados como mediana (rango intercuartílico) y número (proporciones) para datos categóricos.

Los p-valores para la comparación de grupos se han calculado con el test U-Mann Whitney, Ji cuadrado o test exacto de Fischer.

En la tabla II se observa que un 32\% (272) de los trabajadores, estaba expuesto en el momento actual o anterior, pero siempre durante el periodo estudiado, al menos a un agente cancerígeno laboral, de los cuales el 75\% (204) eran varones y el 25\% (68) mujeres. En el grupo de las mujeres trabajadoras los factores de riesgo a los que están más 
expuestas son a los riesgos biológicos $(18,6 \%)$ y radiaciones ionizantes $(4,7 \%)$. Sin embargo los trabajadores varones presentan exposición a los disolventes $(13,6 \%)$ y la sílice $(9,4 \%)$.

Tabla II. Exposición a factores de riesgo cancerígeno en la actividad laboral

\begin{tabular}{|c|c|c|c|c|}
\hline Cancerígenos laborales & $\begin{array}{c}\text { Global } \\
\mathrm{n}=272\end{array}$ & $\begin{array}{c}\text { Mujeres } \\
n=68\end{array}$ & $\begin{array}{l}\text { Varones } \\
\mathrm{n}=204\end{array}$ & P valor \\
\hline Sílice, $\mathrm{n}^{\circ}(\%)$ & $48(5,7)$ & $0(0,0)$ & $48(9,4)$ & $<0,001$ \\
\hline Amianto, $n .^{\circ}(\%)$ & $17(2,0)$ & $0(0,0)$ & $17(3,3)$ & $<0,001$ \\
\hline Plaguicidas, n. $^{\circ}(\%)$ & $40(4,8)$ & $1(0,3)$ & $39(7,6)$ & $<0,001$ \\
\hline Agentes citostáticos n. ${ }^{\circ}(\%)$ & $3(0,4)$ & $2(0,6)$ & $1(0,2)$ & 0,563 \\
\hline Plomo, n. ${ }^{\circ}(\%)$ & $7(0,8)$ & $0(0,0)$ & $7(1,4)$ & 0,048 \\
\hline Radiaciones ionizantes $n .^{\circ}(\%)$ & $27(3,2)$ & $15(4,7)$ & $12(2,3)$ & 0,072 \\
\hline Biológicos (Hepatitis B o C), n. $^{\circ}(\%)$ & $72(8,6)$ & $60(18,6)$ & $12(2,3)$ & $<0,001$ \\
\hline Cloruro de vinilo monómero, $\mathrm{n}^{\circ}(\%)$ & $13(1,6)$ & $1(0,3)$ & $12(2,3)$ & 0,021 \\
\hline Disolventes, n. $^{\circ}(\%)$ & $72(8,6)$ & $2(0,6)$ & $70(13,6)$ & $<0,001$ \\
\hline Óxido de etileno, $\mathrm{n}^{\circ}(\%)$ & $0(0,0)$ & $0(0,0)$ & $0(0,0)$ & - \\
\hline
\end{tabular}

Los valores han sido expresados como número (porcentaje sobre expuestos) para datos categóricos.

Los p-valores para la comparación de grupos se han calculado con el test Ji cuadrado o test exacto de Fischer.

En la tabla III se observan los porcentajes de exposición a cancerígenos por sector de actividad y por sexo. En el sector industria, destaca una alta exposición de los trabajadores varones a disolventes $(21,7 \%)$ en comparación con las mujeres $(1,3 \%)$ $(\mathrm{p}<0,001)$, mientras que la principal exposición de las mujeres en dicho sector es a riesgos biológicos $(17,5 \%)$ frente al $1,2 \%$ de los varones $(p<0,001)$ ). No hallamos ninguna exposición de las mujeres en los sectores de agricultura y ganadería y construcción. Los mayores porcentajes de los trabajadores varones expuestos $(70,8 \%$ y $70,8 \%)$, los hallamos en el sector de agricultura y ganadería frente a plaguicidas y disolventes respectivamente. 
Tabla III. Exposición a factores de riesgo cancerígeno en el trabajo actual y tiempo de exposición global y por género según el sector de actividad económica

\begin{tabular}{|c|c|c|c|c|}
\hline & $\begin{array}{c}\text { Global } \\
\mathrm{n}=272\end{array}$ & $\begin{array}{c}\text { Mujeres } \\
n=68\end{array}$ & $\begin{array}{l}\text { Varones } \\
\mathrm{n}=204\end{array}$ & $\mathbf{P}$ valor \\
\hline Agricultura y Ganadería & $\mathbf{n}=\mathbf{2 5}$ & $\mathbf{n}=\mathbf{1}$ & $\mathrm{n}=24$ & \\
\hline Amianto, $\mathrm{n} \%$ & $2(8,0)$ & $0(0,0)$ & $2(8,3)$ & 0,920 \\
\hline Plaguicidas, n \% & $17(68,0)$ & $0(0,0)$ & $17(70,8)$ & 0,320 \\
\hline Disolventes, n \% & $1(4,0)$ & $0(0,0)$ & $17(70,8)$ & 0,960 \\
\hline Construcción & $n=106$ & $\mathbf{n}=\mathbf{2}$ & $\mathrm{n}=104$ & \\
\hline Sílice, n \% & $14(13,2)$ & $0(0,0)$ & $14(13,5)$ & 0,752 \\
\hline Amianto, $\mathrm{n} \%$ & $7(6,6)$ & $0(0,0)$ & $7(6,7)$ & 0,872 \\
\hline Plaguicidas, n \% & $1(0,9)$ & $0(0,0)$ & $1(1,0)$ & 0,981 \\
\hline Plomo, n \% & $5(4,7)$ & $0(0,0)$ & $5(4,8)$ & 0,907 \\
\hline Cloruro de vinilo monómero, $\mathrm{n} \%$ & $2(1,9)$ & $0(0,0)$ & $2(1,9)$ & 0,962 \\
\hline Disolventes, $\mathrm{n} \%$ & $11(10,4)$ & $0(0,0)$ & $11(10,6)$ & 0,802 \\
\hline Industria & $\mathrm{n}=338$ & $\mathbf{n}=\mathbf{8 0}$ & $\mathrm{n}=\mathbf{2 5 8}$ & \\
\hline Sílice, n \% & $27(8,0)$ & $0(0,0)$ & $27(10,5)$ & 0,001 \\
\hline Amianto, $\mathrm{n} \%$ & $5(1,5)$ & $0(0,0)$ & $5(1,9)$ & 0,596 \\
\hline Plaguicidas, n \% & $17(5,0)$ & $0(0,0)$ & $17(6,6)$ & 0,016 \\
\hline Plomo, n \% & $1(0,3)$ & $0(0,0)$ & $1(0,4)$ & 0,763 \\
\hline Radiaciones ionizantes, $\mathrm{n} \%$ & $7(2,1)$ & $1(1,3)$ & $6(2,3)$ & 0,477 \\
\hline Biológicos (Hep. B o C), n \% & $17(5,0)$ & $14(17,5)$ & $3(1,2)$ & $<0,001$ \\
\hline Cloruro de vinilo monómero, $\mathrm{n} \%$ & $6(1,8)$ & $0(0,0)$ & $6(2,3)$ & 0,342 \\
\hline Disolventes, $\mathrm{n} \%$ & $57(16,9)$ & $1(1,3)$ & $56(21,7)$ & $<0,001$ \\
\hline Servicios & $n=366$ & $\mathrm{n}=239$ & $\mathrm{n}=\mathbf{1 2 7}$ & \\
\hline Sílice, n \% & $7(1,9)$ & $0(0,0)$ & $7(5,5)$ & 0,001 \\
\hline Amianto, $\mathrm{n} \%$ & $3(0,8)$ & $0(0,0)$ & $3(2,4)$ & 0,041 \\
\hline Plaguicidas, n \% & $5(1,4)$ & $1(0,4)$ & $4(3,1)$ & 0,051 \\
\hline Agentes citostáticos, $\mathrm{n} \%$ & $3(0,8)$ & $2(0,8)$ & $1(0,8)$ & 0,723 \\
\hline Plomo, $\mathrm{n} \%$ & $1(0,3)$ & $0(0,0)$ & $1(0,8)$ & 0,347 \\
\hline Radiaciones ionizantes, $\mathrm{n} \%$ & $20(5,5)$ & $14(5,9)$ & $6(4,7)$ & 0,810 \\
\hline Biológicos (Hep. B o C), n \% & $55(15,0)$ & $46(19,2)$ & $9(7,1)$ & 0,002 \\
\hline Cloruro de vinilo monómero, $\mathrm{n} \%$ & $5(1,4)$ & $1(0,4)$ & $4(3,1)$ & 0,051 \\
\hline Disolventes, $\mathrm{n} \%$ & $3(0,8)$ & $1(0,4)$ & $2(1,6)$ & 0,277 \\
\hline
\end{tabular}

Los valores han sido expresados como número (porcentaje) para datos categóricos.

Los p-valores para la comparación de grupos se han calculado con el test Ji cuadrado o test exacto de Fischer.

Los trabajadores que más fuman, por sectores de actividad, son los que pertenecen a los sectores servicios $(55,3 \%)$ y al sector industria $(41,1 \%)$, siendo los trabajadores del sector agricultura y ganadería, los que en global refieren un menor hábito de fumar (12\%). Respecto al factor exposición, los porcentajes de los trabajadores pertenecientes al grupo de los no expuestos, presentan cifras más elevadas de consumo de tabaco en todos los sectores de actividad (Tabla IV). 
Tabla IV. Consumo de tabaco por sector de actividad económica

\begin{tabular}{|c|c|c|c|c|}
\hline Consumo de tabaco & $\begin{array}{c}\text { Global } \\
\mathrm{n}=835\end{array}$ & $\begin{array}{c}\text { No Expuestos } \\
n=563\end{array}$ & $\begin{array}{c}\text { Expuestos } \\
\mathrm{n}=\mathbf{2 7 2}\end{array}$ & $P$ valor \\
\hline Agricultura y Ganadería & $\mathrm{n}=\mathbf{2 5}$ & $\mathbf{n}=\mathbf{8}$ & $\mathbf{n}=\mathbf{1 7}$ & \\
\hline No fumador, $\mathrm{n}^{\circ}(\%)$ & $15(60,0)$ & $5(62,5)$ & $10(58,8)$ & \multirow{3}{*}{0,484} \\
\hline Exfumador, $\mathrm{n}^{\circ}(\%)$ & $7(28,0)$ & $4(50,0)$ & $3(17,6)$ & \\
\hline Fumador, n. ${ }^{\circ}(\%)$ & $3(12,0)$ & $3(37,5)$ & $0(0,0)$ & \\
\hline Construcción & $n=106$ & $n=67$ & $\mathbf{n}=\mathbf{3 9}$ & \\
\hline No fumador, $\mathrm{n}^{\circ}(\%)$ & $29(27,4)$ & $19(28,4)$ & $10(25,6)$ & \multirow{3}{*}{0,320} \\
\hline Exfumador, $\mathrm{n}^{\circ}(\%)$ & $50(47,1)$ & $15(22,3)$ & $35(89,7)$ & \\
\hline Fumador , n. ${ }^{\circ}(\%)$ & $27(25,4)$ & $22(32,8)$ & $5(12,8)$ & \\
\hline Industria & $\mathrm{n}=338$ & $\mathbf{n}=\mathbf{2 0 7}$ & $\mathbf{n}=\mathbf{1 3 1}$ & \\
\hline No fumador, $\mathrm{n}^{\circ}(\%)$ & $81(23,9)$ & $35(16,9)$ & $46(35,1)$ & \multirow{3}{*}{0,028} \\
\hline Exfumador, $\mathrm{n}^{\circ}(\%)$ & $118(34,9)$ & $48(23,1)$ & $70(53,4)$ & \\
\hline Fumador, n. $^{\circ}(\%)$ & $139(41,1)$ & $100(48,3)$ & $39(29,7)$ & \\
\hline Servicios & $\mathrm{n}=336$ & $\mathrm{n}=\mathbf{2 8 1}$ & $\mathrm{n}=85$ & \\
\hline No fumador, $\mathrm{n}^{\circ}(\%)$ & $60(17,8)$ & $40(14,2)$ & $20(23,5)$ & \multirow{3}{*}{0,681} \\
\hline Exfumador, n. ${ }^{\circ}(\%)$ & $90(26,8)$ & $55(19,5)$ & $35(41,1)$ & \\
\hline Fumador, n. $^{\circ}(\%)$ & $186(55,3)$ & $148(52,6)$ & $38(44,7)$ & \\
\hline
\end{tabular}

Los valores han sido expresados como número (porcentaje) para datos categóricos.

Los p-valores para la comparación de grupos se han calculado con el test Ji cuadrado.

El mayor porcentaje de trabajadores de nuestro estudio, que refiere tener un patrón de consumo de alcohol habitual (52\%), pertenece a los trabajadores del sector de la agricultura y ganadería, sector en el que también se encuentra el porcentaje más alto de trabajadores expuestos con un consumo habitual de alcohol (58,8\%). En global, los trabajadores del sector servicios, son los que menos refieren consumir alcohol de forma habitual (15,3\%) (Tabla V). 
Tabla V. Consumo de Alcohol por sector de actividad económica

\begin{tabular}{|c|c|c|c|c|}
\hline Consumo de Alcohol & $\begin{array}{c}\text { Global } \\
\mathrm{n}=835\end{array}$ & $\begin{array}{c}\text { No Expuestos } \\
\mathbf{n}=563\end{array}$ & $\begin{array}{c}\text { Expuestos } \\
\mathrm{n}=\mathbf{2 7 2}\end{array}$ & $P$ valor \\
\hline Agricultura y Ganadería & $\mathrm{n}=\mathbf{2 5}$ & $\mathbf{n}=\mathbf{8}$ & $\mathbf{n}=\mathbf{1 7}$ & \\
\hline No consume, n. $^{\circ}(\%)$ & $7(28,0)$ & $4(50,0)$ & $3(17,6)$ & \multirow{4}{*}{0,239} \\
\hline Consumidor anterior, n. $^{\circ}(\%)$ & $3(12,0)$ & $0(0,0)$ & $3(17,6)$ & \\
\hline Consumidor ocasional, $n .^{\circ}(\%)$ & $2(8,0)$ & $1(12,5)$ & $1(5,9)$ & \\
\hline Consumidor habitual, n. $^{\circ}(\%)$ & $13(52,0)$ & $3(37,5)$ & $10(58,8)$ & \\
\hline Construcción & $n=106$ & $n=67$ & $\mathrm{n}=39$ & \\
\hline No consume, $\mathrm{n}^{\circ}(\%)$ & $14(13,2)$ & $7(10,4)$ & $7(17,9)$ & \multirow{4}{*}{0,317} \\
\hline Consumidor anterior, $\mathrm{n}^{\circ}(\%)$ & $16(15,1)$ & $8(11,9)$ & $8(20,5)$ & \\
\hline Consumidor ocasional, $n .^{\circ}(\%)$ & $39(36,8)$ & $28(41,8)$ & $11(28,2)$ & \\
\hline Consumidor habitual, n. $^{\circ}(\%)$ & $37(34,9)$ & $24(35,8)$ & $13(33,3)$ & \\
\hline Industria & $\mathrm{n}=338$ & $\mathrm{n}=\mathbf{2 0 7}$ & $\mathrm{n}=131$ & \\
\hline No consume, . $^{\circ}(\%)$ & $92(27,2)$ & $60(29,0)$ & $32(24,4)$ & \multirow{4}{*}{0,034} \\
\hline Consumidor anterior, $\mathrm{n}^{\circ}(\%)$ & $73(21,6)$ & $48(23,2)$ & $25(19,1)$ & \\
\hline Consumidor ocasional, $n .^{\circ}(\%)$ & $107(31,7)$ & $69(33,3)$ & $38(29,0)$ & \\
\hline Consumidor habitual, n. $^{\circ}(\%)$ & $66(19,5)$ & $30(14,5)$ & $36(27,5)$ & \\
\hline Servicios & $\mathrm{n}=336$ & $\mathrm{n}=\mathbf{2 8 1}$ & $\mathrm{n}=85$ & \\
\hline No consume, n. $^{\circ}(\%)$ & $158(43,2)$ & $114(40,6)$ & $44(51,8)$ & \multirow{4}{*}{0,231} \\
\hline Consumidor anterior, $\mathrm{n}^{\circ}(\%)$ & $79(21,6)$ & $64(22,8)$ & $15(17,6)$ & \\
\hline Consumidor ocasional, $n .^{\circ}(\%)$ & $73(19,9)$ & $56(19,9)$ & $17(20,0)$ & \\
\hline Consumidor habitual, n. $^{\circ}(\%)$ & $56(15,3)$ & $47(16,7)$ & $9(10,6)$ & \\
\hline
\end{tabular}

Los valores han sido expresados como número (porcentaje) para datos categóricos.

Los p-valores para la comparación de grupos se han calculado con el test Ji cuadrado.

En global, la proporción más baja de sujetos activos (35,7\%), así como la menor proporción de realización de una dieta equilibrada $(42,2 \%)$, figuran en el sector servicios. En relación al factor exposición, dentro del grupo de los no expuestos, el porcentaje de trabajadores que más refieren llevar a cabo una dieta equilibrada $(73,4 \%)$, pertenecen al sector industria, mientras que en el grupo de los expuestos, el mayor porcentaje (70,6\%), corresponde al sector de agricultura y ganadería (Tabla VI).

Tabla VI. Dieta y actividad física por sector de actividad económica

\begin{tabular}{|c|c|c|c|c|}
\hline Dieta y actividad física & $\begin{array}{l}\text { Global } \\
\mathrm{n}=835\end{array}$ & $\begin{array}{c}\text { No Expuestos } \\
n=563\end{array}$ & $\begin{array}{c}\text { Expuestos } \\
n=272\end{array}$ & $P$ valor \\
\hline Agricultura y Ganadería & $\mathrm{n}=\mathbf{2 5}$ & $\mathbf{n}=\mathbf{8}$ & $\mathbf{n}=\mathbf{1 7}$ & \\
\hline Activos, n. $^{\circ}(\%)$ & $16(64,0)$ & $6(75,0)$ & $10(58,8)$ & 0,661 \\
\hline Dieta equilibrada, $n .^{\circ}(\%)$ & $17(68,0)$ & $5(62,5)$ & $12(70,6)$ & 0,513 \\
\hline Construcción & $\mathrm{n}=106$ & $\mathrm{n}=67$ & $\mathrm{n}=39$ & \\
\hline Activos, n. $^{\circ}(\%)$ & $43(40,6)$ & $28(41,8)$ & $15(38,5)$ & 0,838 \\
\hline Dieta equilibrada, n. $^{\circ}(\%)$ & $62(58,5)$ & $25(37,3)$ & $19(48,7)$ & 0,308 \\
\hline Industria & $\mathrm{n}=338$ & $\mathbf{n}=\mathbf{2 0 7}$ & $\mathrm{n}=\mathbf{1 3 1}$ & \\
\hline Activos, $\mathrm{n}^{\circ}(\%)$ & $153(45,2)$ & $89(42,9)$ & $64(48,8)$ & 0,545 \\
\hline Dieta equilibrada, $n .^{\circ}(\%)$ & $228(67,5)$ & $152(73,4)$ & $76(58,0)$ & 0,004 \\
\hline Servicios & $\mathrm{n}=336$ & $\mathrm{n}=\mathbf{2 8 1}$ & $\mathrm{n}=\mathbf{8 5}$ & \\
\hline Activos, n. $^{\circ}(\%)$ & $120(35,7)$ & $98(34,8)$ & $22(25,8)$ & 0,036 \\
\hline Dieta equilibrada, $n .^{\circ}(\%)$ & $142(42,2)$ & $118(41,9)$ & $24(28,2)$ & 0,006 \\
\hline
\end{tabular}

Los valores han sido expresados como número (porcentaje) para datos categóricos.

Los p-valores para la comparación de grupos se han calculado con el test Ji cuadrado o test exacto de Fischer. 


\section{DISCUSIÓN}

Según este estudio, los datos referentes a la distribución por sexo de la población trabajadora son de un $61 \%$ varones y $39 \%$ mujeres. Estos resultados difieren ligeramente de los datos que figuran en la EPA $2011^{13}$, cuyos resultados fueron un $55,5 \%$ varones y $44,5 \%$ mujeres. Pensamos que esta pequeña diferencia podría estar relacionada con la existencia de contratos eventuales y con jornadas parciales, que se realizan más frecuentemente a las mujeres trabajadoras ${ }^{13}$, y que pese a estar cubiertas por algún servicio de prevención ajeno, en bastantes ocasiones no se les ha ofrecido la posibilidad de la realización de su correspondiente examen de salud laboral, no estando incluidas por lo tanto en nuestra población de estudio.

La realización de la jornada laboral de los trabajadores de nuestro estudio, es de forma mayoritaria mediante jornada partida (mañana y tarde) y turnicidad. Estos datos son congruentes con los resultados publicados en la segunda encuesta sobre Condiciones de Trabajo en las empresas de Castilla y León ${ }^{14}$. Este aspecto podría estar influenciado, en que como se ha expuesto anteriormente, la mayoría de la población ocupada de la provincia de Salamanca, pertenece a los sectores de actividad de servicios e industria, sectores en los que predominan dichos tipos de jornada laboral.

Según los resultados hallados en este estudio, un 32\% de la población trabajadora estuvo expuesta durante el periodo estudiado, al menos a un agente cancerígeno laboral. La falta de datos empíricos sobre los niveles de exposición de los trabajadores españoles, a los diferentes agentes cancerígenos en las distintas ocupaciones y actividades económicas, así como por provincias o comunidades autónomas, impide conocer con precisión estos niveles, teniendo que recurrir a estudios de ámbito nacional como el denominado Carex-Esp ${ }^{15}$, realizado por el Dr. Manolis Kogevinas y publicado en 2006, en el que se analizan las exposiciones a cancerígenos laborales de la población trabajadora en el año 2004. Al comparar los resultados de nuestro trabajo con los encontrados en Carex-Esp ${ }^{15}$, observamos que la exposición global a agentes cancerígenos laborales en la provincia de Salamanca $(32,5 \%)$ es superior a la exposición a nivel nacional $(25,4 \%)$.

Los agentes cancerígenos más frecuentes a los que estaba expuesta la población trabajadora de este estudio, riesgos biológicos (Hepatitis $\mathrm{B}$ o $\mathrm{C}$ ), disolventes y sílice, también difieren en parte con los resultados encontrados en el estudio Carex-Esp ${ }^{15}$, en el que los agentes más frecuentes fueron la radiación solar, sílice y humo de tabaco ambiental.

Las discordancias existentes entre este estudio y el estudio Carex-Esp ${ }^{15}$, pueden deberse a distintos motivos:

- La cantidad de trabajadores expuestos a radiación solar, que figuraba como la exposición más frecuente a cancerígenos en el estudio Carex-Esp ${ }^{15}$, sería actualmente muy distinta, debido en parte a que la fecha de realización de dicho estudio fue anterior al inicio de la grave crisis económica que afecta a España en los últimos años, fundamentalmente a partir del año 2008, influyendo de forma directa en una fuerte reducción del número de trabajadores del sector de la construcción, sector clave precisamente en la realización de tareas a la intemperie, con la consiguiente exposición de trabajadores ante la radiación solar.

- La presencia del humo del tabaco ambiental como principal exposición a cancerígenos en los trabajadores, en estudios como el Carex-Esp ${ }^{15}$ o el llevado a cabo por Janer G. y cols. ${ }^{16}$, realizados con anterioridad a la entrada en vigor en España de la Ley 42/2010 de 30 de diciembre $^{17}$ (ley antitabaco), no serían de aplicación a la población laboral actual, existiendo una disminución muy importante ante dicha exposición debido a la ampliación de la prohibición de fumar a la práctica totalidad de espacios públicos cerrados, incluidos bares y restaurantes, afectando de forma directa a la exposición de un importante número de trabajadores fundamentalmente del sector servicios, situación que se traduciría en una escasa exposición actual de los trabajadores a el humo de tabaco ambiental. 
El mayor consumo de tabaco por los trabajadores de este estudio se produce en el sector servicios, seguido de los sectores industria y construcción, hallando en el sector de agricultura y ganadería el menor número de fumadores. Estos datos son concordantes con los resultados obtenidos en un estudio reciente ${ }^{18}$, sobre la prevalencia de consumo de tabaco en la población trabajadora tras la entrada en vigor de la Ley 42/2010 ${ }^{17}$, en el cuál también se advierte un descenso progresivo del porcentaje de fumadores, afectando de forma directa a los trabajadores de la hostelería, circunstancia que puede haber influido en que la cifra de fumadores de nuestro estudio, dentro del sector servicios, hubiese sido aún mayor. El ambiente laboral ha sido, hasta la aprobación de la Ley $28 / 2005^{19}$, uno de los espacios en los que se producía con mayor intensidad el tabaquismo pasivo.

Los trabajadores no expuestos a cancerígenos laborales en este estudio fuman más que los trabajadores expuestos. La explicación más plausible de este hallazgo es que los trabajadores expuestos a cancerígenos laborales tienen una mayor concienciación sobre la conveniencia de abandonar el hábito tabáquico, debido a que ya tienen un riesgo perjudicial para su salud derivado de su actividad laboral, y a que gracias a los cursos y asesoramiento que reciben por parte de los equipos de Medicina del Trabajo, pertenecientes a sus servicios de prevención ajenos, son conocedores de que el hábito de fumar puede aumentar el efecto nocivo de ciertos cancerígenos laborales, como la exposición al amianto, sílice, ciertos plaguicidas y disolventes.

Otro hallazgo interesante en este estudio en relación con el consumo de tabaco, es la presencia mayoritaria de mujeres en edad fértil dentro del sector servicios, sector donde los trabajadores referían un mayor consumo de tabaco, con los posibles riesgos derivados para la gestación y para la descendencia, datos coincidentes con el estudio llevado a cabo por Vicente Herrero M.T. ${ }^{20}$ sobre la situación actual del tabaquismo entre los trabajadores de correos España tras la Ley 28/2005 ${ }^{19}$. Esta circunstancia puede estar influida por la presencia de estrés, ya que muchas mujeres siguen teniendo que ocuparse totalmente de las responsabilidades domésticas además de mantener una vida laboral activa. Estos factores domésticos unidos a aspectos laborales como la distribución de horarios y carga de trabajo, pueden aumentan la probabilidad de que sean fumadoras.

El consumo de alcohol en este estudio, es superior en los trabajadores varones que en las mujeres, siendo mayoritario su consumo en los sectores de agricultura y ganadería y construcción. Los trabajadores expuestos a cancerígenos laborales son los que refieren un consumo de alcohol mayor, más frecuente y en mayor cantidad en comparación con los trabajadores no expuestos. Estos datos coinciden con la información obtenida de la encuesta de consumo de sustancias psicoactivas en el ámbito laboral en España ${ }^{21}$. En esta encuesta también se refleja como factor influyente, la realización de trabajos peligrosos o en condiciones penosas (frío, calor, posturas incómodas), condiciones que mayoritariamente se suelen presentar en la realización de tareas precisamente de los sectores de agricultura y ganadería y construcción.

Los trabajadores de los sectores industria y servicios de este estudio, sometidos a mayor turnicidad, tienen las cifras más bajas de consumo del alcohol en comparación al resto de sectores de actividad. Si bien es cierto que en estudios anteriores ${ }^{22,23}$ se consideraba la existencia de rotaciones de turno durante la jornada laboral, como uno de los múltiples factores de riesgo laborales posibles para presentar problemas relacionados con el alcohol, un estudio reciente ${ }^{24}$, demuestra que trabajadores de doble turno presentan una menor prevalencia en el consumo de alcohol respecto a trabajadores de jornadas diurnas.

Los trabajadores que realizan menos actividad física y que menos refieren llevar a cabo una dieta equilibrada en este estudio, son los que pertenecen al sector servicios, datos que coinciden con los de un estudio realizado sobre actitudes de los trabajadores frente a los programas de promoción de la salud para la prevención del cáncer en el lugar del trabajo ${ }^{25}$, en el cuál se analizan entre otros aspectos, las actitudes hacia los programas de promoción de la salud, en una muestra compuesta fundamentalmente por trabajadores del sector servicios (hostelería, personal sanitario de un hospital, ayuntamiento y 
universidad) de Cataluña y Mallorca, obteniendo como parte de los resultados, que sólo un $45,5 \%$ de los trabajadores refieren llevar a cabo una dieta equilibrada, y que apenas la mitad de los trabajadores $(51,5 \%)$ refiere realizar ejercicio físico de forma habitual fuera del lugar de trabajo.

\section{CONCLUSIONES}

Tras la revisión de la literatura científica, este es el primer estudio que analiza los hábitos de vida de los trabajadores en relación a su exposición a cancerígenos en el trabajo.

Los resultados muestran como el consumo de tabaco es superior en los trabajadores expuestos a algún cancerígeno laboral, sin embargo los trabajadores no expuestos, refieren consumir más alcohol de forma habitual y realizar de forma más frecuente una dieta equilibrada. La realización de actividad física habitual es similar en los dos grupos de trabajadores.

Como limitaciones de este estudio, cabe señalar que al tratarse de un estudio transversal nos impide estimar relaciones de asociación causal. Otra limitación que puede influir, es que el volumen de realización de exámenes de salud laboral de cada servicio de prevención ajeno, utilizado en la selección de trabajadores de nuestro estudio, puede variar cada año en función de los contratos y productos concertados que estos servicios de prevención tengan con las empresas.

A pesar de las limitaciones mencionadas y haciendo una interpretación prudente de los resultados, este trabajo permite extraer conclusiones globales y cumple el propósito para el cuál fue diseñado, pues proporciona información para futuras intervenciones en la prevención del cáncer laboral, teniendo en cuenta que obviamente, la exposición a cancerígenos varía en España según las comunidades autónomas, en función del tipo de industria y tejido empresarial de cada zona.

\section{BIBLIOGRAFÍA}

1. Botteri E, Iodice S, Raimondi S, Maisonneuve P, Lowenfels AB. Cigarette smoking and adenomatous polyps: a meta-analysis. Gastroenterology. 2008 Feb; 134(2):388-95.

2. Ribes J, Cleries R, Esteban L, Moreno V, Bosch FX. The influence of alcohol consumption and hepatitis B and $\mathrm{C}$ infections on the risk of liver cancer in Europe. J Hepatol. 2008 Aug;49 (2): 233-42.

3. Observatorio Español sobre Drogas. Encuesta 2007-2008 sobre el consumo de sustancias psicoactivas en el ámbito laboral en España. Madrid: Ministerio de Sanidad, Política Social e Igualdad. Secretaría General de Política Social y Consumo. Delegación del Gobierno para el Plan Nacional Sobre Drogas; 2011. Disponible en: http://www.pnsd.msc.es/Categoria2/publica/pdf/InformeDrogasEmergentes.pdf.

4. Aguilar Cordero MJ, González Jiménez E, García López AP, Álvarez Ferre J, Padilla López CA, Guisado Barrilao R, et al. Obesity and its implication in breast cancer. Nutr Hosp. 2011 Jul-Aug; 26(4): 899-903.

5. Fernández Rodríguez M. et al. Valoración nutricional de trabajadores sanitarios expuestos a turnicidad en Canarias. Nutr. Hosp. [revista en internet]. 2004 [consultado 15 de diciembre de 2013];5(19):286-291. Disponible en: http://scielo.isciii.es/scielo.php?script=sci_arttext\&pid=S021216112004000500007\&lng=e s\&nrm=iso.

6. Díaz Sampedro E, López Maza R, González Puente M. Hábitos de alimentación y actividad física según la turnicidad de los trabajadores de un hospital. Enferm Clin. 2010; 20(4): 229-235.

7. Díaz Franco J. Estrés alimentario y salud laboral vs. estrés laboral y alimentación equilibrada. Med. segur trab. [revista en internet]. 2007 [consultado 20 de diciembre de 2013]:53(209):93-99. Disponible en: $<$ http://scielo.isciii.es/scielo.php?script=sci_arttext\&pid=S0465546X2007000400012\&lng=es\&nrm=iso $>$. http://dx.doi.org/10.4321/S0465-546X2007000400012.

8. Matos $\mathrm{CH}$, Pacheco Da Costa R, Costa S. Trabajo en producción de comidas: consecuencias en la alimentación y estado nutricional de los trabajadores. Med. segur. trab. [revista en internet]. 2009 [consultado 20 de diciembre de 2013]:55(214):91-100. Disponible en: http://scielo.isciii.es/scielo. php?script=sci_arttext $\&$ pid $=$ S0 465546X2009000100008\&lng=es\&nrm $=$ iso 
9. Kogevinas M, Maqueda J, De la Orden V, Fernández F, Kauppinen T, Benavides F. Exposición a carcinógenos laborales en España: aplicación de la base de datos CAREX. Arch Prev Riesgos Labor. 2000; 3(4):153-59.

10. Real Decreto 39/1997, de 17 de enero, por el que se aprueba el Reglamento de los Servicios de Prevención. Boletín Oficial del Estado, no 27 (31 de enero de 1997).

11. IARC Working Group on the Evaluation of Carcinogenic Risks to Humans. International Agency for Research in Cancer monographs on the evaluation of carcinogenic risks to humans and their supplements: A complete list. IARC Monogr Eval Carcinog Risks Hum. Lyon, France. [monografía en internet]. 19722005 [acceso 20 de siembre de 2013];1-88 Disponible en: http://monographs.iarc.fr/ENG/Classification/ index.php

12. Real Decreto Legislativo $1 / 1995$, de 24 de marzo, por el que se aprueba el texto refundido de la Ley del Estatuto de los Trabajadores. Boletín Oficial del Estado, $n^{\circ} 75$ (1 de Mayo de 1995).

13. Encuesta de Población Activa (2011). Datos para el cuarto trimestre de 2011. [acceso 28 de diciembre de 2013] Disponible en: http://www.ine.es/prensa/epa_prensa.htm

14. Junta de Castilla y León, Consejería de Economía y Empleo. II Encuesta sobre Condiciones de Trabajo en las empresas de Castilla y León. Valladolid: Junta de Castilla y León, Consejería de Economía y Empleo; 2010.

15. Kogevinas M, Rudolf VH. Carex-Esp: Sistema de Información sobre Exposición Ocupacional a Cancerígenos en España en el año 2004. [consultado 28 de diciembre de 2013]. Disponible en: http:// hesa.etuirehs.org/uk/newsevents/files/InformeCarex.pdf.

16. Janer G, Sala M, Kogevinas M. Health promotion trials at worksites and risk factors for cancer. Scandinavian journal of work, environment \& health. 2002:141-57.

17. Ley $42 / 2010$, de 30 de diciembre, por la que se modifica la Ley $28 / 2005$, de 26 de diciembre, de medidas sanitarias frente al tabaquismo y reguladora de la venta, el suministro, el consumo y la publicidad de los productos del tabaco. Boletín Oficial del Estado n 318 (31 de diciembre de 2010).

18. Catalina Romero C, Sainz Gutiérrez JC, Quevedo Aguado L, Cortés Arcas MV, Pinto Blázquez JA, Gelpi Méndez JA, et al. Prevalencia de consumo de tabaco en población trabajadora tras la entrada en vigor de la ley 42/2010. Revista española de salud pública. 2012; 86(2): 177-88.

19. Ley $28 / 2005$, de 26 de diciembre, de medidas sanitarias frente al tabaquismo y reguladora de la venta, el suministro, el consumo y la publicidad de los productos del tabaco. Boletín Oficial del Estado $n^{\circ} 309$ (27 de diciembre de 2005).

20. Vicente-Herrero MT, López-González A, Santamaría-Navarro C, de Paz Merchán S, Rodríguez-Fernández R. Situación actual del tabaquismo entre los trabajadores de correos-España tras la Ley 28/2005. Medicina y Seguridad del Trabajo. 2008; 54(213): 87-95.

21. Observatorio Español sobre Drogas. Encuesta 2007-2008 sobre el consumo de sustancias psicoactivas en el ámbito laboral en España. Madrid: Ministerio de Sanidad, Política Social e Igualdad. Secretaría General de Política Social y Consumo. Delegación del Gobierno para el Plan Nacional Sobre Drogas; 2011.

22. Navarro J, Megías E, Gómez E. La incidencia de las drogas en el medio laboral de la Comunidad Valenciana. Fundación de Ayuda contra la Drogadicción y Generalitat Valenciana Madrid. 1999.

23. Ochoa Mangado E, Madoz Gúrpide A. Consumo de alcohol y otras drogas en el medio laboral. Medicina y Seguridad del Trabajo. 2008; 54(213): 25-32.

24. Ramírez Sánchez DC, Martínez Barroso KA, Asúnsolo del Barco Á. Abuso de alcohol en el medio laboral, factores de riesgo para el consumo, e instrumentos de valoración aplicables en la vigilancia de la salud. Medicina y Seguridad del Trabajo. 2011; 57(224): 190-209.

25. Sala M, Janer G, Font C, Garau I, Sole MD, Corbella T, et al. [Employees attitudes to workplace health promotion programs for cancer prevention]. Gac Sanit. 2002 Nov-Dec; 16(6): 521-5. 